\title{
Electrophysiological studies in the post-viral fatigue syndrome
}

\author{
GORAN A JAMAL, STIG HANSEN
}

From the Glasgow University Department of Neurology, Institute of Neurological Sciences, Southern General Hospital and West of Scotland Health Boards Department of Clinical Physics and Bio-engineering, Glasgow, Scotland

SUMMARY Single fibre electromyography (SFEMG) was studied in 40 patients with the post-viral fatigue syndrome. These patients were also assessed clinically, serologically, virologically and immunologically. About $75 \%$ of the patients had definitely abnormal SFEMG results. This was regarded as evidence of abnormality in the peripheral part of the motor unit. The muscle fibre was the likely site of involvement.

The post-viral fatigue syndrome, also known as myalgic encephalomyelitis, has been recognised recently as a distinct neurological entity with increasing evidence of the organic nature of the disease. ${ }^{1}$ Most cases occur in epidemics but it may be endemic and sporadic cases may occur.' The cardinal clinical features are of a benign encephalomyelitis combined with emotional disturbance, myalgia and muscle fatigue. The latter is one of the most constant features.' Muscle weakness, wasting and fasciculations with retention of normal reflexes $^{2}$ and increased urinary creatine output ${ }^{34}$ occur in few cases.

Several electrophysiological studies have failed to demonstrate definite abnormality of any part of the motor unit. ${ }^{56}$ However, reduced recruitment pattern of voluntary motor units was seen and some patients, particularly during recovery, showed "grouping" of motor units on maximal voluntary contraction. ${ }^{2}$ These findings were nonspecific and regarded by some as not organic. ${ }^{7}$ We applied the single fibre electromyographic (SFEMG) technique developed by Ekstedt and Stalberg ${ }^{8-10}$ to study the pathophysiology of this disease.

\section{Patients and methods}

Forty patients were included in this study. Their ages

Address for reprint requests: Dr Goran A Jamal, Glasgow University Department of Neurology, Institute of Neurological Sciences, Southem General Hospital, Glasgow, G51 4TF, Scotland.

Received 28 September 1984. Accepted 29 October 1984 ranged between $17-55$ years with a mean of 38.8 years (SD 11.2 years). Twenty four were female $(60 \%)$ and 16 male $(40 \%)$. The duration of the illness, ranged from 3 months to more than 20 years (mean 5 years).

A preceding viral infection was documented in all the patients: in three cases with varicella, in two with rubella and in the remainder with one of the coxsackie $B$ viruses. All the patients had gross fatigue as their primary symptom. This was present at rest but made worse by exercise. Full neurological examination was negative in all and no muscle weakness was demonstrated in any patient. Routine laboratory tests (urinanalysis, ESR, full blood count, urea and electrolytes, liver function tests and muscle enzymes), chest radiographs and ECG were normal. In none, was anti-acetylcholine antibody found.

The $\mathbf{4 0}$ patients were subjected to a variety of serological, virological and immunological studies. In 20 patients, muscle biopsy was performed and was examined by routine histological, histochemical and ultrastructural methods. The results of these and other clinical data are described elsewhere." ${ }^{31} \mathrm{P}$ nuclear magnetic resonance (NMR) study on muscle was also carried out on a patient from this series and the result was published separately. ${ }^{12}$

Prior to the neurophysiological tests, a careful clinical history was taken with particular emphasis on present or past history of cervical radiculopathy, neuropathy, nerve trauma, diabetes mellitus and any other concurrent problem in the neuromuscular system. In addition alcohol ingestion was assessed and a drug history taken. Routine sensory and motor conduction studies were performed on all patients using conventional surface recordings in the right common peroneal, right median and right sural nerves. ${ }^{13}$ These results were compared with those of 32 normal subjects whose age ranged between 16-57 (mean $=38 \cdot 2, \mathrm{SD}=13 \cdot 9$ ) years. Normal conduction studies were prerequisite for inclusion of the patient in the study. 
Concentric needle electromyography (CNEMG) was performed on several muscles in each patient to exclude evidence of neuropathy or myopathy. Repetitive stimulation of the right ulnar nerve was also performed to exclude a gross neuromuscular transmission defect. In five patients with the most abnormal SFEMG jitter, the repetitive stimulation study was repeated after regional curarisation with normal results.

Single fibre EMG was performed on the right exensor digitorum communis muscle irrespective of the patient's disability. This muscle was used because of minimal age related changes below 60 years, ${ }^{14}$ small standard deviation of jitter values and the availability of several control studies. ${ }^{1415}$

A standard Medelec SFEMG needle with a leading off surface of $25 \mu \mathrm{m}$ diameter was used and recordings were made using a Hewlett Packard electromyograph feeding a "jitter unit" where controls allowed selection of level triggers to record suitable fast rise points on the action potentials. Output pulses synchronous with these points were fed on line to a PD12 computer with a real time clock. This incremented every $2.5 \mu \mathrm{s}$ and thus allowed measurement of the interval between a pair of single fibre potentials. This method contributes at most an error of $1.2 \mu \mathrm{s}$ to the jitter measurement. Up to 500 potential pairs could be acquired in this way and were initially displayed on a monitor as a graph of the sequential interpotential intervals. Editing of spurious points was then performed before calculating the following. (1) the percentage of impulse blocking. (2) mean interdischarge interval, giving a measure of firing rate. (3) mean inter-potential interval for the group. This was in all our patients between $0.5-1.5 \mathrm{~ms}$. (4) mean consecutive difference which represents the jitter value. (5) mean sorted data difference. The significance of the latter and the previously mentioned parameters has been reviewed elsewhere..$^{1016}$

The SFEMG needle was inserted into the muscle whilst the patient maintained a slight voluntary contraction. The needle was manipulated until a pair of potentials was obtained, both of which fulfilled the criteria of single muscle fibre potential. ${ }^{8}$ Care was taken to maintain a steady recruitment rate of 8-15 impulses per second. In each patient five or six recordings of potential pairs were obtained.

The normal jitter for this muscle is $23.4 \mu$ s with a standard deviation of $8.5 \mu \mathrm{s} .^{9}$ This gives an upper limit of normal (99\% confidence limit) at $55 \mu$ s for any single potential pair. Two values greater than $55 \mu$ s has been regarded as abnormal in the context of 20 potential pairs that is, $10 \% .^{16}$ The mean overall jitter is another index of jitter and this has been shown to have an upper normal limit of $34 \mu \mathrm{s} .{ }^{15}$ We have used a very strict definition of abnormality in this study which is least likely to give false positive results - that is, two pairs of the five studied (40\%) greater than $55 \mu$ s or one pair greater than $55 \mu$ s and the mean overall jitter of the remaining pairs is more than 34 $\mu s$. A mean overall jitter greater than $34 \mu$ s with none of the pairs above $55 \mu$ s was regarded as probably abnormal.

\section{Results}

The CNEMG was normal in all the patients apart from non specific recruitmental abnormalities. Grouping phenomena similar to those described in other studies ${ }^{2}$ was seen in 14 patients. The individual units potentials within these groups were normal in duration and amplitude. In 10 patients there was a reduced recruitment pattern without grouping.

Adequate sets of SFEMG recordings were obtained from all the patients. The jitter values for the 40 patients are plotted in the figure. Twenty four patients had two or more pairs with jitter values more than $55 \mu \mathrm{s}$. The mean overall jitter was greater than $34 \mu \mathrm{s}$ in 28 patients. By combining both criteria, $75 \%$ of our patients had definite abnormalities of jitter. One patient was defined as probably abnormal. The mean overall jitter in all our patients were greater than the control mean. We found a number of low jitter values $(<10 \mu \mathrm{s})$ implying muscle fibre splitting. ${ }^{16}$

There was no evidence of impulse or concomitant blocking ${ }^{16}$ in any of the patients with either normal or abnormal jitter values.

\section{Discussion}

Variability of the interpotential intervals of two single fibre potentials belonging to the same motor unit "that is, the jitter" can only arise where there are two pathways for the initially single discharge to follow. Therefore, any abnormality proximal to the branching of the motor axon cannot be implicated in abnormal jitter. The source of jitter would therefore be from one or more of the following: the terminal nerve twigs, the endplate or the muscle fibre.

Impulse blocking usually occurs at jitter values exceeding $80-100 \mu \mathrm{s}$ in the extensor digitonum communis muscle in cases of neuromuscular transmission defect as in myasthenia gravis, after curarisation or in cases of neuropathy due to sprouting and immature endplate formation with transmission defects. ${ }^{16}$ Absence of blocking in our patients despite marked increases in the jitter values probably makes the neuromuscular junction an unlikely site of involvement. Concomitant blocking, ${ }^{17}$ which none of our patients showed, indicates unreliable axonal impulse conduction. Therefore, it is reasonable to assume that the site of involvement is less likely to be the axonal twigs or the neuromuscular junction and it is probably due to a disturbed muscle fibre membrane conduction. It is well known that increased jitter may be due to disturbed conduction along the muscle fibre membrane in certain pathological conditions. ${ }^{1618}$ Disuse atrophy, neurogenic atrophy and primary muscle disease such as polymyositis can alter the propagation velocity in the muscle fibre membrane and SFEMG abnor- 


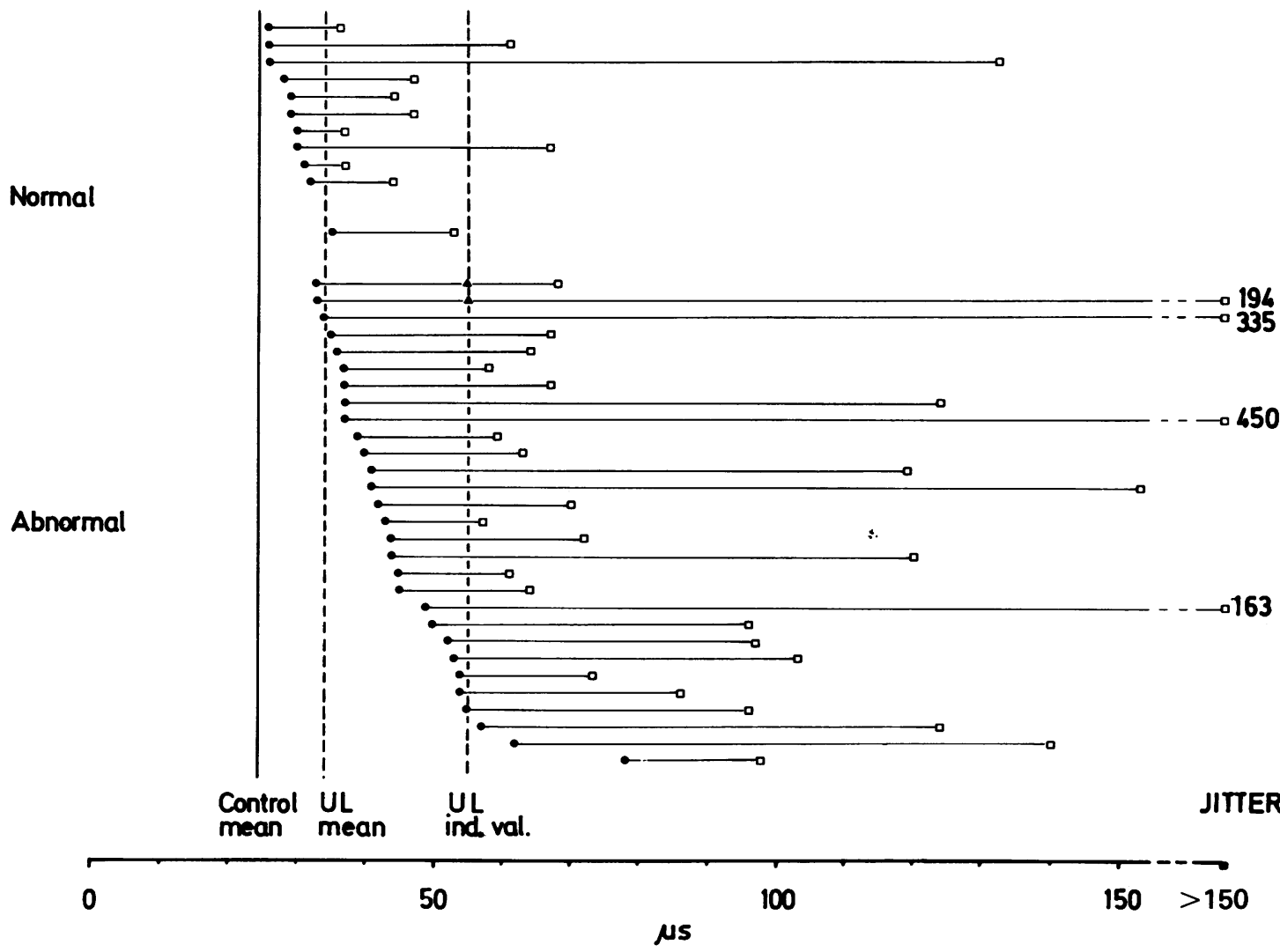

Fig Jitter value for 40 patients with the post-viral fatigue syndrome. Each patient is represented by a horizontal line. The $\operatorname{dot}(\bullet)$ at the left end of the line represents the mean overall jitter excluding the pair with the highest jitter. The squares ( $\square$ ) at the right end of the line represent the pair with the highest jitter. The second highest values for two patients are represented by triangles $(\Delta)$ indicating that they have abnormal jitter because of two pairs with values greater than $55 \mu s$. Control values are indicated by vertical lines signifying (from left to right): Mean jitter (23.4 $\mu \mathrm{s})$, the upper limit of mean overall jitter (34 $\mu \mathrm{s})$ and the upper limit of jitter for a single pair (55 $\mathrm{\mu s})$.

malities have been observed in the latter condition. ${ }^{1920}$ Schiller et al reported increased jitter values in patients with viral infection having myalgia in the acute phase. ${ }^{21}$

It has been suggested that a metabolic disorder in the muscle fibre, due to damage of some intracellular enzymes caused by persistent virus infection, occurs in the post viral fatigue syndrome. ${ }^{.1}{ }^{31} \mathrm{P}$ NMR examination of a case from this series with high jitter values demonstrated abnormal muscle metabolism in the form of excessive and early intracellular acidosis during exercise. ${ }^{12}$ Muscle biopsies were abnormal in all the 20 cases examined from this series. The most important findings were type II fibre predominance, subtle and scattered muscle fibre necrosis and bizarre tubular structures and mitochondrial abnormalities." Our findings of few very low jitter values is consistent with the presence of scattered muscle fibre necrosis seen in muscle biopsy.

We conclude that we have shown clear electrophysiological evidence of an abnormality in the peripheral part of the motor unit in patients with post viral fatigue syndrome. This abnormality has been shown in patients not suffering from neuropathy or myopathy by clincal, laboratory and electrophysiological criteria. At present, involvement of the muscle fibre membrane seem an attractive hypothesis to explain the abnormalities reported.

We thank Dr PO Behan for permission to study the patients under his care. We are also grateful to Professor JA Simpson and Dr AI Weir for continuous support and helpful criticism. 


\section{References}

${ }^{1}$ Editorial. Epidemic myalgic encephalomyelitis. $\mathrm{Br}$ Med J 1978;1:1436-7.

${ }^{2}$ Richardson AT. Some aspects of the Royal Free Hospital epidemic. Ann Phys Med 1956;3:81-9.

${ }^{3}$ White DN, Burtch RB. Iceland disease: A new infection simulating acute anterior poliomyelitis. Neurology (Mineap) 1954;4:506-16.

4 Albrecht RM, Oliver VL, Poskanzer DC. Epidemic neuromyasthenia, outbreak in a convent in New York State. JAMA 1964;187:904-7.

${ }^{5}$ Bauwens P. Electrodiagnosis in motor unit dysfunction. Proc $R$ Soc Med 1955;48:194-7.

- Alexander SJ. Observations on neuromuscular dysfunction in the Addingtoin outbreak. S Afr Med J 1956;30: 88-90.

${ }^{7}$ McEvedy CP, Beard AW. Royal Free epidemic of 1955: A reconsideration. $\mathrm{Br}$ Med J 1970;1:7-11.

${ }^{8}$ Ekstedt J. Human single muscle fibre action potentials. Acta Physiol Scand, Supplement 226 1964;61:1-96.

' Stalberg E, Ekstedt J, Broman A. The electromyographic jitter in normal human muscles. Electroencephalalogr Clin Neurophysiol 1971;31:429-38.

${ }^{10}$ Eksteoit J, Nilsson G, Stalberg E. Calculation of the electromyographic jitter. J Neurol Neurosurg Psychiatry 1974;37:526-39.

${ }^{11}$ Behan PO, Behan WMH and Bell EJ. The post-viral syndrome. An analysis of the findings in 50 cases. Journal of Infection (in press).

12 Arnold DL, Radda GK, Bore PJ, Styles P, Taylor DJ.
Excessive intracellular acidosis of skeletal muscles on exercise in a patient with a post-viral exhaustion/ fatigue syndrome. Lancet 1984;1:1367-9.

${ }^{13}$ Lenman JAR, Ritchie AE. Clinical Electromyography. 2nd Ed, Bath: Pitman. 1977:60-72.

${ }^{14}$ Stalberg E, Thiele B. Motor unit fibre density in extensor digitorum communis muscle. J Neurol Neurosurg Psychiatry 1975;38:874-80.

15 Sanders DB, Howard JF, Johns TR. Single fiber electromyography in myasthenia gravis. Neurol (Minneap) 1979;29:68-76.

${ }^{16}$ Stalberg E, Trontelji JV. Single Fibre Electromyography. Old Woking, Mirvalle Press Ltd. 1979.

${ }^{17}$ Stalberg E, Thiele B. Transmission block in terminal nerve twigs: a single fibre electromyographic finding in man. J Neurol Neurosurg Psychiatry 1972;35:52-9.

${ }^{18}$ Stalberg E, Ekstedt J. Single fibre electromyography and microphysiology of the motor unit in normal and disease human muscle. In: Desmedt JE ed. New Developments in Electromyography and Clinical Neurophysiology. Vol 1. Basel: Karger. 1973;1:11329.

${ }^{19}$ Stalberg E. Propagation velocity in human muscle fibre in situ. Acta Physiol Scand, Supplement 287 1966;70:7-112.

${ }^{20}$ Henriksson KG, Stalberg E. The terminal innervation pattern in polymyositis: A histochemical and single fibre EMG study. Muscle Nerve 1978;1:3-13.

${ }^{21}$ Schiller HH, Schwartz MS, Friman G. Disturbed neuromuscular transmission in viral infection. New Engl J Med 1977;296:884. 